\title{
Daily exercise alters gene expression profiles in the nucleus tractus solitarii of the spontaneously hypertensive rat; Novel mechanisms underlying exercise-induced cardiovascular adaptation?
}

\author{
Hidefumi Waki
}

Department of Physiology, Wakayama Medical University School of Medicine

It is generally accepted that a regular moderate aerobic exercise is recommended as a non-drug treatment to improve and prevent essential hypertension. However, the mechanisms underlying exercise-induced hypotensive effects remain poorly understood. With evidence that sympathetic nervous activity was attenuated by exercise therapy in hypertensive patients (1), we proposed that brain mechanisms are involved in the therapeutic effects on blood pressure. Whilst numerous brainstem regions can control sympathetic nerve activity, the nucleus tractus solitarii (NTS) is a most attractive central region since it plays a pivotal role in the regulation of set-point of blood pressure (2). Moreover, the NTS is known as a pivotal region in the central network, integrating the baroreceptor sensory information with other inputs such as muscle afferents, making it an ideal site for integrating cardiovascular responses to exercise (3). Thus, we propose a role for the NTS in the cardiovascular adaptation induced by chronic daily exercise. In the present study, we have investigated whether alterations of genetic characteristics at the level of the NTS could contribute to exercise-induced cardiovascular adaptation. We have performed a microarray study to screen for genes in this nucleus that are differentially expressed between the spontaneously hypertensive rats (SHR) assigned to daily voluntary exercise group (SHR-Ex, from 4 to 16 weeks of age, $n=6)$ and those assigned to sedentary group $(n=6)$. Total RNA from the NTS of each animal was extracted and Rat Genome Oligo DNA Microarray (Agilent Technologies, Inc.) was performed. Pathway analysis by KEGG database (http://www.kegg.jp) revealed that exercise therapy in the SHR altered the expression levels of NTS genes which are functionally associated with neuroactive ligand-receptor interaction, cell adhesion molecules, cytokine-cytokine receptor interaction, or Jak-STAT signaling pathway. One of those genes was histamine H1 receptor which was down-regulated in the NTS of SHR-Ex. To identify the functional roles of H1 receptors within the NTS on cardiovascular regulation, we microinjected $\mathrm{H} 1$ receptor agonist, 2-pyridylethylamine dihydrochloride, into the NTS of anaesthetized rats $(n=6)$. When the agonist was bilaterally microinjected, blood pressure was significantly increased, indicating that H1 receptors within the NTS may play an important role for regulating the set point of blood pressure.We conclude that daily exercise in the SHR alters gene expression profiles at the level of the NTS although mechanisms underlying these alterations have yet to be elucidated. It is also suggested that a down-regulation of $\mathrm{H} 1$ receptor gene within the NTS may be involved in the exercise-induced cardiovascular adaptation. The study was financially supported by the JSPS (21300253) and the Takeda Science Foundation. References(1) Izdebska E, et al. J Physiol Pharmacol. 2004; 55: 713-724. (2) Waki H, et al. Hypertension. 2007; 49: 1321-1327. (3) Potts JT. Exp Physiol. 2006; 91: 59-72. 